\title{
Characterization of Hantavirus $\mathbf{N}$ protein intracellular dynam- ics and localization
}

\author{
Robert-William Welke ${ }^{1, t}$, Hannah Sabeth Sperber ${ }^{2, t}$, Amit Koikkarah $^{3}$, Laura Menke ${ }^{4}$, Christian Sieben ${ }^{4,5}$, Detlev H. \\ Krüger ${ }^{6}$, Salvatore Chiantia ${ }^{7}$, Andreas Herrmannn ${ }^{1,8}$, Roland Schwarzer ${ }^{2 *}$
}

1 Department of Molecular Biophysics, Humboldt University, Berlin, Germany

2 Institute for Translational HIV Research, University Hospital Essen, Essen, Germany

3 University of Potsdam, Institute of Biochemistry and Biology, Potsdam, Germany

4 Nanoscale Infection Biology Group, Department of Cell Biology, Helmholtz Centre for Infection Research, Braunschweig, Germany

5 Institute for Genetics, Technische Universität Braunschweig, Braunschweig, Germany

6 Institut für Virologie, Charité - Universitätsmedizin Berlin, Gliedkörperschaft der Freien Universität Berlin und der Humboldt-Universität zu Berlin, Berlin, Germany

7 University of Potsdam, Institute of Biochemistry and Biology, Potsdam, Germany

8 Biophysikalische Chemie, Freie Universität, Berlin, Germany

* Correspondence: Roland-Schwarzer@gmx.de

+ These authors contributed equally to this work.

\begin{abstract}
Hantaviruses are enveloped viruses that possess a tri-segmented, negative-sense RNA genome. The viral S-segment encodes the multifunctional nucleocapsid protein $(\mathrm{N})$, which is involved in genome packaging, intracellular protein transport, immunoregulation and several other crucial processes during hantavirus infection.

In this study we have generated fluorescently tagged $\mathrm{N}$ protein constructs derived from $\mathrm{Pu}$ umalavirus, the dominant hantavirus species in Central, Northern and Eastern Europe. We have comprehensively characterized this protein in the rodent cell line $\mathrm{CHO}-\mathrm{K} 1$, monitoring the dynamics of $\mathrm{N}$ protein complex formation and investigating co-localization with host proteins as well as the viral glycoproteins Gc and Gn. We found a significant spatial correlation of N with vimentin, actin and P-bodies, but not with microtubules. N constructs also co-localized with $\mathrm{Gn}$ and Gc, albeit not as strong as the glycoproteins associated with each other. Moreover, we assessed oligomerization of $\mathrm{N}$ constructs, observing efficient and concentration-dependent multimerization, with complexes comprising more than 10 individual proteins.
\end{abstract}

Keywords: Hantavirus; N protein; oligomerization; actin; P-Bodies; vimentin; Number\&Brightness; Puumalavirus; macromolecular assemblies

\section{Introduction}

Hantaviruses (HV, order Bunyaviridae) are a family of emerging viruses, causing life threatening human zoonoses with case fatalities of up to $60 \%$ [1,2]. In Europe the less virulent hantavirus species Puumala (PUUV) causes the majority of reported hantavirus-associated diseases.

Puumala virus particles comprise a lipid envelope and a single-stranded, trisegmented RNA genome that encodes for five proteins [3]: the nucleocapsid protein N, the glycoproteins Gn and Gc, an RNA dependent RNA polymerase RdRp, and the non-structural protein NSs. Entry of HV particles into their target cell, predominantly of the endothelial lineage [4], is mediated by the viral spike complex, a Gc/Gn heterotetramer. We and others have shown that, after engagement with their receptor, Old world hantaviruses exploit multiple entry routes to get access to the vulnerable host cell cytoplasm [5-7]. Subsequent virus replication takes place at the ER-Golgi intermediate compartment (ERGIC) 
and involves $\mathrm{N}$ - and RdRp-mediated cap-snatching as a prerequisite of viral translation [8]. It has been proposed that, throughout the course of an infection, viral factories are formed, which involves ER and Golgi membranes, P-bodies and ribosomes as well as multiple cytoskeleton components [6,8,9]. Virus assembly and budding is then mediated by the three structural proteins, Gc, Gn and N. The latter binds the viral RNA, thus forming ribonucleoprotein complexes which eventually recruit the virus genome to the nascent virion $[10,11]$. Virus particle formation on the other hand is believed to be solely controlled by the HV glycoproteins Gc and Gn. Ultimately, Gn and Gc interact with both the vRNA and the associated $\mathrm{N}$ proteins [12,13], leading to the formation of mature virions [6]. After budding into the ERGIC, virus particles are then released from the infected cell by mechanisms that are barely understood [6,14]. Throughout the course of an infection cycle hantaviruses trigger a substantial reorganization of the cytoskeleton and overall structural organization of their host cells $[5,15,16]$, however the underlying processes and involved host-pathogen interactions remained cryptic.

This study focuses on one of the key structural proteins of PUUV, the nucleoprotein $\mathrm{N}$. We have generated chimeric proteins that harbor fluorescent proteins fused to the Nterminus of $\mathrm{N}$, to be able to assess the dynamics of $\mathrm{N}$ localization and trafficking in livecell experiments. We found that N-YFP rapidly clusters in expressing cells, eventually forming macromolecular complexes that can extend through most of the cell body. We also observed preferential co-localization with P-bodies, actin and vimentin, but not tubulin, suggesting selective association with cytoskeleton components. Upon co-expression with other structural PUUV proteins, the glycoproteins Gc and Gn, strong spatial correlation was found in the perinuclear region, likely indicative of nascent virus assembly processes. Finally, using in fluorescence fluctuation spectroscopy experiments we observed large scale oligomerization of N-YFP, which did not markedly change when viral glycoproteins were co-expressed.

Our experiments aimed at studying properties and intracellular activities of the PUUV N protein, independent of infections and largely in the absence of most other viral proteins and viral genomic RNA. Our goal was to explore inherent properties of $\mathrm{N}$ that govern its intracellular localization and processing, which eventually contributes to PUUV particle formation and release. The data therefore shed new light on the intricate interplay between cellular and viral components, which could reveal key vulnerabilities of hantavirus infection cycles.

\section{Materials and Methods}

Generation of fluorescently labelled Hantavirus N protein. Vero E6 cells were infected with the Puumalavirus, strain Sotkamo (V-2969/81), an Orthohantavirus from the family of the Hantaviridae. At day 3 post infection, cells were subjected to mRNA extraction (RNeasy, Qiagen) and reverse transcription. Then, cDNA was used for amplification via PCR using primers for PUUV N protein. $\mathrm{N}$ gene PCR products were cloned into pmYFP-N1 vectors including A206K monomeric mutation of the fluorescent tag using NotI and BsrgI restriction sites. An additional Turquoise construct was generated by excising mYFP using AgeI and NotI restriction sites and replacing it with a Turquoise PCR product.

Cell culture and transfection. Chinese hamster ovary (CHO-K1) cells and African green monkey kidney epithelial cells (Vero E6 cells) were maintained in Dulbecco's modified Eagle's medium (DMEM, PAA Laboratories GmbH, Austria) containing 10\% fetal bovine serum. 24-48 hours prior to experiments, fusion protein expression plasmids were transfected into cell lines in $35 \mathrm{~mm}$ Glass Bottom Microwell Dishes (MatTek Corporation) utilizing Turbofect (Thermo Scientific, Waltham, MA, USA) according to the manufacturer's protocol. All cell lines other than CHO-K1 (Figure S2) were seeded in a standard 12 -well tissue culture plate (Greiner) on $18 \mathrm{~mm}$ glass cover slips (\#1.5, Menzel). The cells were seeded at a density of 150.000 cells per well and cultivated in DMEM with $10 \%$ fetal calf serum (Sigma). After $24 \mathrm{~h}$, the cells were transfected with $1 \mu \mathrm{g}$ plasmid DNA per well 
using jetOptimus (Polyplus) transfection reagent. The cells were further incubated for 24h, then fixed, stained and mounted on microcopy glass slides (ProLong Gold, ThermoFisher).

Immunofluorescence staining. For intracellular immunofluorescence staining, cells were washed three times with phosphate-buffered saline with calcium and magnesium (PBS+/+) and fixed with 3.7\% paraformaldehyde for $25 \mathrm{~min}$ at room temperature. Afterwards, the cells were washed three times with PBS+/+ before permeabilization with $0.2 \%$ Triton X-100 and $0.2 \%$ bovine serum albumin (BSA) for $20 \mathrm{~min}$. After three more washing steps, cells were incubated with primary antibodies for $1 \mathrm{~h}$ at room temperature (RT), followed by three washing steps and another incubation with conjugated secondary antibody for $1 \mathrm{~h}$ at RT. Finally, cells were washed three more times and subjected to microscopy. All cell lines other than CHO-K1 (Figure S2) were fixed, stained and mounted on microcopy glass slides (ProLong Gold, ThermoFisher) 24h post transfection. Immunostaining was performed as described using anti-tubulin antibodies (B512, Sigma) and Phalloidin-Alexa647 (ThermoFisher) at a concentration of $0.16 \mu \mathrm{M}$.

Fluorescence microscopy. Confocal spinning disc microscopy (CSD) was used for all antibody stains and long-term exposure experiments. Images were acquired with a Visitron VisiScope scanning-disc confocal laser microscope (Visitron Systems, Puchheim, Germany) and $a \times 60 / 1.2$ UPlanSApo water or a $\times 100 / 1.3$ UPlanFLN oil objective (pixel size of 0.13 and $0.2 \mu \mathrm{m}$, respectively). An Andor iXon 888 EMCCD camera $(1024 \times 1024$ pixels, Andor, Belfast, Northern Ireland) was used to detect fluorescence. Excitation was carried out using the following diode lasers: $488 \mathrm{~nm}$ (FITC) with an ET525/50-nm emission filter, $561 \mathrm{~nm}$ (Atto550) with an ET600/50-nm emission filter, 640 nm (PCA635P) with an ET700/75-nm emission filter and $405 \mathrm{~nm}$ (DAPI) with an ET460/50-nm emission filter. If not otherwise mentioned, z-stacks were acquired, and z-projections were displayed and analyzed. N-YFP single stains and co-transfection experiments were imaged using an Olympus FluoView 1000MPE confocal microscope, equipped with 60x/1.2 Water (UPlanSApo), and 60x/1.45 Oil (UPlanSApo) objective respectively, as well as $405 \mathrm{~nm}, 440 \mathrm{~nm}$, $561 \mathrm{~nm}$ and $635 \mathrm{~nm}$ diode laser and $488 \mathrm{~nm}, 515 \mathrm{~nm}$ Argon lasers with the following filter sets: $80 / 20,405-458 / 515 / 559 / 635,405 / 488 / 559 / 635,458 / 515,405 / 458 / 515$. All cell lines other than CHO-K1 (Figure S2) were imaged using a Nikon Ti2 spinning disk confocal microscope.

Table 1: Reagents for fluorescence and immunofluorescence staining.

\begin{tabular}{|l|l|}
\hline Primary Antibodies & Manufacturer \\
\hline Hantavirus (Puumala) N protein antibody & Fitzgerald, UK (cat. 10R-2502) \\
\hline Anti-Vimentin antibody & Abcam, UK (cat. ab45939) \\
\hline Anti-Dcp1a antibody & Abcam, UK (cat. ab57654) \\
\hline Anti-tubulin (clone B512) & Sigma-Aldrich, USA (cat. T5168) \\
\hline Fluorescently conjugated phalloidin & Thermo Fisher Scientific (cat. R415) \\
\hline Secondary Antibodies & Manufacturer \\
\hline AlexaFluor 488 goat anti rabbit IgG (H+L) & Invitrogen, USA (cat. A-11008) \\
\hline AlexaFluor 488 goat anti mouse IgG (H+L) & Abcam, UK (cat. ab150117) \\
\hline AlexaFluor 568 goat anti mouse IgG (H+L) & Invitrogen, USA (cat. A-11004) \\
\hline Alexa fluor 594 goat anti rabbit IgG (H+L) & Invitrogen, USA (cat. A-11012) \\
\hline Alexa fluor 647 goat anti mouse IgG (H+L) & Invitrogen, USA (cat. A-21235) \\
\hline Alexa fluor 647 goat anti rabbit IgG (H+L) & Abcam, UK (cat. ab150087) \\
\hline
\end{tabular}

Image analysis. Manual and semi-automatic image processing and analysis were carried out with ImageJ (https://imagej.nih.gov/ij/). Spot detection (Figure 1) was performed using the ComDet plug-in developed by Eugene Katrukha on maximum-intensity projections of z-stacks. Intensity thresholds were adjusted according to no-virus control samples. The following settings were used for analysis: a particle size of 4 pixels and an 
intensity threshold of 6 . The analysis included but did not segment larger particles. Automatic image analysis was performed with CellProfiler [17] using an in-house pipeline (available upon request). Briefly, cells were identified in the DNA staining channel using the "identify primary objects" module. Then, cell bodies were segmented in either actin, microtubule, vimentin or DIC images using the module "identify secondary objects". Thereafter, the module "MeasureColocalization" was executed to assess the pixel-by-pixel Pearson correlation coefficient.

Number and Brightness analysis. Number and brightness analysis (N\&B) was performed as previously described in Petazzi et al. [18]. Briefly, 3 to 6 x105 cells were plated onto 35-mm glass-bottom dishes (CellVis, CA or MatTek Corp., USA) $48 \mathrm{~h}$ prior to the experiment and transfected with the plasmids of interest. Confocal images were acquired using a Zeiss LSM780 microscope (Carl Zeiss Microscopy GmbH, Germany). The $488 \mathrm{~nm}$ excitation from a CW Argon laser was focused with a 40 UPLS Apochromat 1.2 NA water objective into the sample. The fluorescence signal was collected by a Zeiss QUASAR multichannel GaAsP detector in the 498 to $606 \mathrm{~nm}$ range in photon-counting mode. 128x 128 pixels images were acquired with pixel dimensions of $400 \mathrm{~nm}$ and a pixel dwell time of 25 to $50 \mu \mathrm{s}$. Image time-stacks of 105 scans were collected using the Zeiss Black ZEN software. The intensity time-stacks data were analyzed using a self-written Matlab code (The MathWorks, Natick, MA, USA). The Matlab algorithm utilizes the equations from Digman et al. [19] for obtaining the molecular brightness and number as a function of pixel position. Bleaching and minor cell movements were partially corrected using a boxcar-filter with an 8-frame window, applied pixel-wise, as previously described [20,21]. Final brightness values were calculated by extrapolating the partial brightness values (i.e., calculated within each 8-frame window) to the earliest time point. Detector saturation leading to artefactual reduction in brightness was avoided by excluding pixels with photon-counting rates exceeding $1 \mathrm{MHz}$. Detector response was corrected by taking into account the signal originating from a thin film containing immobilized fluorophores [22]. Each region of interest (ROI) contained ca. 102 pixels and the corresponding brightness values were usually symmetrically distributed around an average value. Finally, the average brightness values obtained from each ROI/cell were normalized to account for the monomer brightness and the fluorescence probability (pm), which summarizes the detectability of the tag [22]. The concentration $\mathrm{N}$ (in monomer units) was calculated by dividing the mean count rate in the ROI by the absolute brightness of the reference monomer. The detailed explanation of the calculation is provided in [18].

Statistical test. To address cell-to-cell variance of the parameter under study, single cells were analyzed separately for quantitative evaluation of microscopy experiments. If not otherwise stated, experimental data represent the mean \pm SEM of individually analyzed cells. Statistical significance was typically assessed using parametric one-way analysis of variance (ANOVA) test and displayed as follows: ${ }^{* * *} \mathrm{P}<0.0001{ }^{* * *} \mathrm{P}<0.001{ }^{* *} \mathrm{P}=$ $0.001-0.01 ;{ }^{*} \mathrm{P}=0.01-0.05$.

\section{Results}

3.1 Generation and characterization of fluorescently tagged $N$ protein constructs. To be able to investigate $\mathrm{N}$ protein dynamics using live fluorescence microscopy we have designed fusion proteins, consisting of a N-terminal yellow fluorescent protein (YFP) and the ORF of the PUUV N protein, obtained from cDNAs of in vitro-infected VeroE6 cells. First, this construct, henceforward termed N-YFP, was transfected into CHO-K1 cells in order to provide an initial, unbiased investigation of its overall, intracellular distribution and expression kinetics. Then, we imaged cells at $24 \mathrm{~h}$ post transfection (p.t.), thus obtaining a general overview of N-YFP expression pattern at a steady-state among multiple cells. Of note, we found a broad variety of cells with highly diverse intracellular distributions of N-YFP (Figure 1A). Whereas some cells exhibited a punctate expression pattern, others showed fibrillar spikes or arch-like structures extending through most of the cell body 
(Figure 1A, blue arrows). To ensure that the observed N-YFP distributions are not solely artifacts caused by the N-terminal tagging of the viral protein, we also transfected nontagged $\mathrm{N}$ protein variants into $\mathrm{CHO}-\mathrm{K} 1$ cells, followed by immunofluorescence staining and imaging, which revealed similar heterogenous structures, including the previously described punctate, fibrillar and arch-like assemblies (Figure S1A).

3.2 Dynamics of N-YFP aggregate formation. Next, we sought to investigate N-YFP clustering kinetics and dynamics. To this aim, we transfected CHO-K1 cells with N-YFP and monitored N-YFP expression by time-lapse live microscopy for extended periods of time. Interestingly, we observed that N-YFP clustering typically starts in both, the perinuclear region and the cell periphery and then extends into other cytoplasmic regions until most of the cell body harbors N-YFP clusters or larger aggregates (Figure 1B, SI Figure S1A). We then imaged several cells for up to 12.5 hours p.t. and measured N-YFP aggregation and overall expression by quantitative image analysis using the ImageJ plugin ComDet. Of note, N-YFP expression levels initially increased steadily, before reaching a maximum at roughly 10 hours p.t. (Figure $1 \mathrm{C}$ solid line, SI Figure S1B). In contrast, average cluster-size and fluorescence intensity reached saturation levels already at 5-7 hours p.t. (Figure 1C, scatter plots). The respective standard deviations, however, further increased throughout the entire observation period (SI Figure S1C). This finding indicates that N-YFP aggregates with average size (which represent the majority of all NYFP clusters) are formed at early time points p.t., preceding the protein's maximum, steady-state expression at the later stages. This notion is further supported by the observation that the cluster number reaches a maximum at $5.5 \mathrm{~h}$ p.t. and declines thereafter (Figure 1C, dashed line).
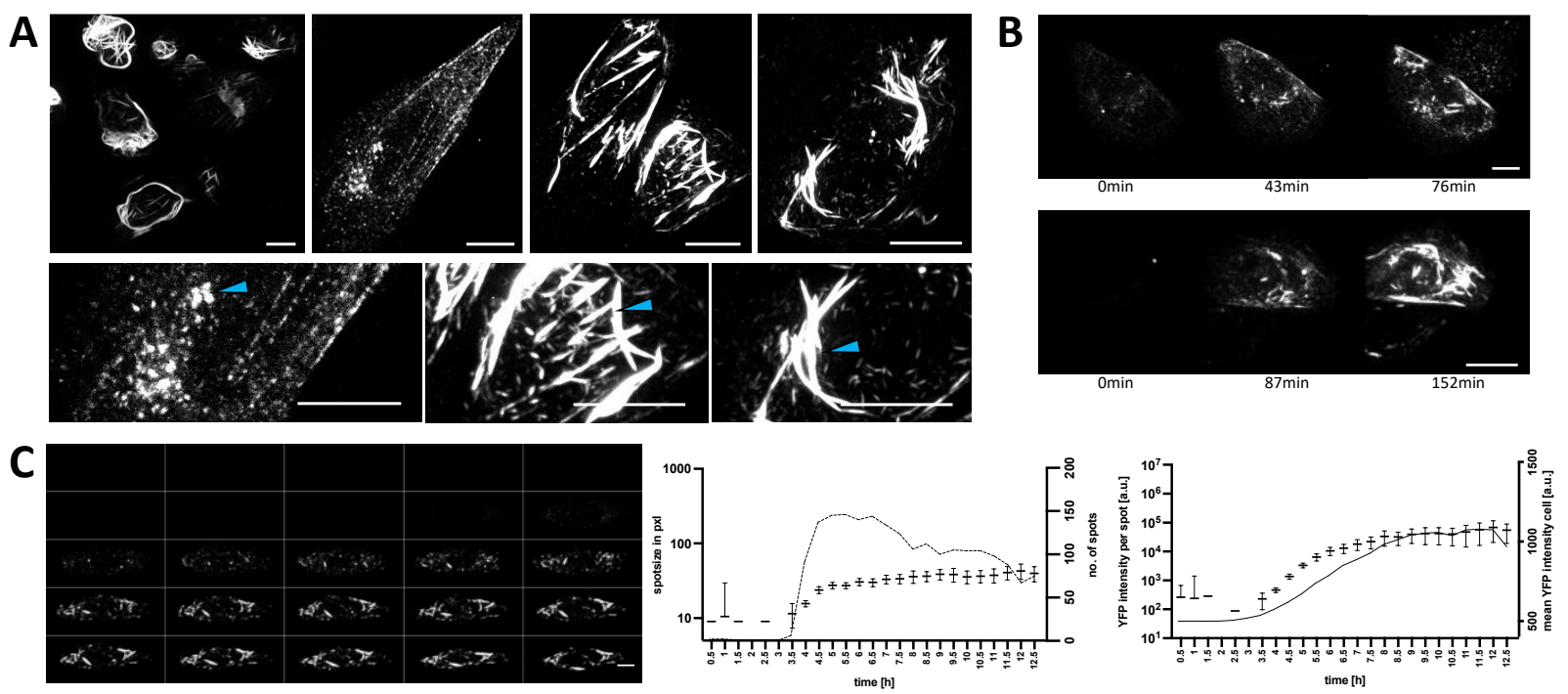

Figure 1: Heterogeneity and dynamics of N-YFP expression patterns. (A) CHO-K1 cells were transfected with N-YFP for $24 \mathrm{~h}$ and observed via spinning disk microscopy. Transfected cells show highly diverse and heterogenous distributions of N-YFP throughout most of the cell body. The image on the upper left shows an overview of multiple cells. Individual examples are shown on the right and magnified in the lower section of the panel. Blue arrows highlight specific protein aggregation states (punctate, spikes and arch-like tubules). (B) The dynamics of N-YFP assemblies were observed by live time-lapse microscopy over several hours post transfection. Two example cells are shown: the upper cell shows protein aggregations emanating from the cell periphery, whereas the lower cell exhibited larger cluster assembly starting in the perinuclear region. (C) Representative cell (panel), repeatedly imaged for $12.5 \mathrm{~h}$ post transfection. Each individual micrograph shows the same cell, imaged in 30 minutes intervals. Protein aggregates were identified by automated image analysis using the ImageJ plugin ComDet. The graph on the on the right show size and fluorescence intensity of single protein aggregates plotted over time (grey dots, see SI Figure S1B for additional examples). Bars show the mean with $95 \%$ confidence interval. The solid lines indicate the overall mean YFP-intensities of the entire cell, whereas the dashed line shows the overall number of 
detected spots (both plotted on right $\mathrm{Y}$-axes). All micrographs show maximum intensity projections of z-stacks. Scale bars: $10 \mu \mathrm{m}$.

3.3 N-YFP co-localizes with vimentin and actin fibers. Our initial characterization of N-YFP indicated a preferential cluster formation in the cell periphery, likely lining the plasma membrane. Such distribution is reminiscent of the cortical actin network. Moreover, it was previously reported that different hantaviruses specifically exploit cytoskeleton components, including vimentin and actin [15]. Therefore, to test for an association of N-YFP with the latter two proteins, we performed a specific staining of N-YFP transfected cells using anti-vimentin antibodies and Rhodamine-Phalloidin (actin-staining), respectively (Figure 2A). Of note, N-YFP fibrillar structures often clearly co-localized with actinspikes, a property that was also found in other mammalian cell lines, including the human lung epithelial line A549 and the bank vole derived MGLU-R culture [23] (SI Figure S2A). The vimentin distribution was more diffuse, but nonetheless coincided markedly with NYFP aggregates (Figure 2A). We next sought to quantitatively evaluate of our fluorescence microscopy images and performed multiple line-plots, statistically analyzing correlation between N-YFP and vimentin or actin (Figure 2B, SI Figure S2B-D). Lines were selected so to include significant signal in all 3 channels and as expected, plots demonstrated a strong association between actin and N-YFP fibers, an observation that corroborated our initial observations. Vimentin, being less distinctly arranged in pin-like structures than actin, still showed a fairly high correlation with N-YFP in multiple cells, however with a much higher variability than actin (Figure 2B, SI Figure S2B-D).

A

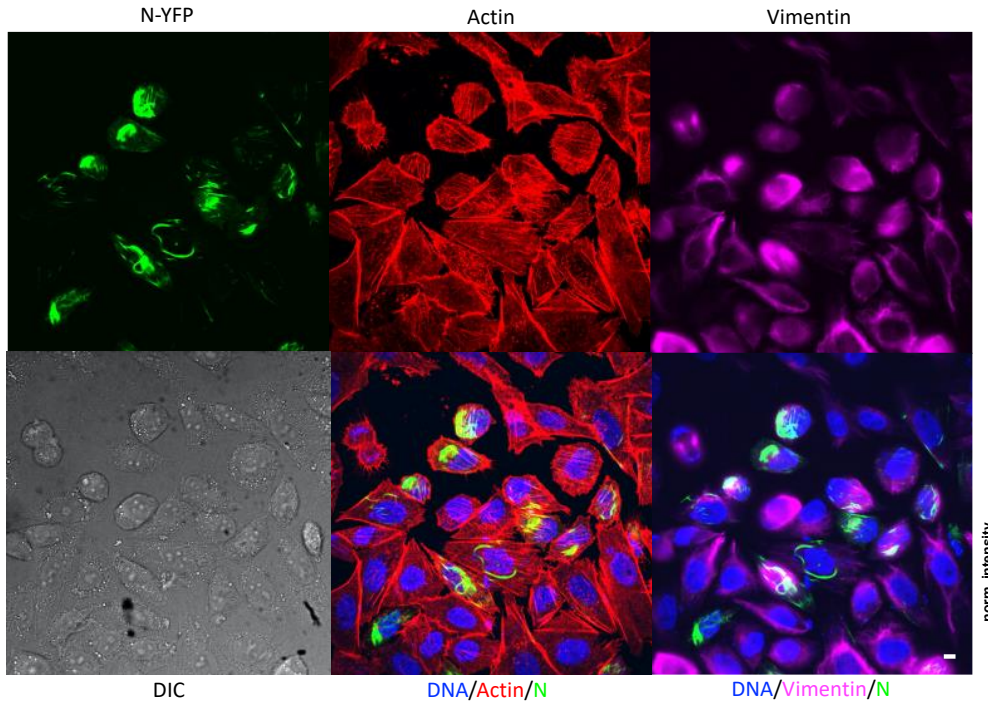

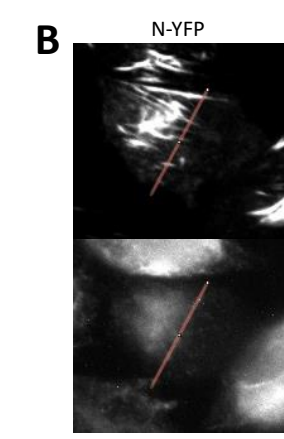

Vimentin

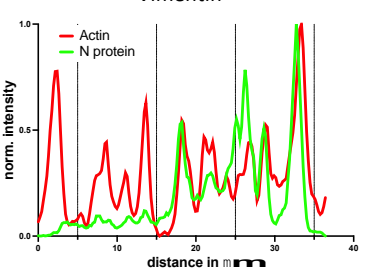

Actin

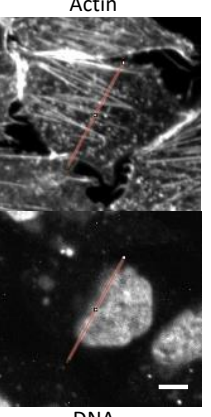

DNA

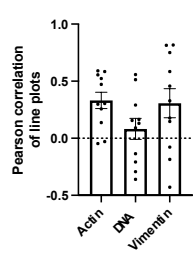

Figure 2: N-YFP co-localizes with cellular actin and vimentin. (A) CHO-K1 cells were transfected with N-YFP and stained for actin using Rhodamine-Phalloidin and vimentin by immunofluorescence. (B) Line-plot analysis of individual cells reveals marked co-localization of N-YFP and actin filaments as well as vimentin. The panel shows one representative cell and the corresponding line plots from all fluorescence channels after normalization. The bar chart on the lower right shows a quantitative analysis of line plots from multiple cells $(n>10)$. Pair-wise Pearson correlations of line plots were calculated for N-YFP with the cellular markers as stated in the bar chart. Bars show mean with SEM. Additional examples can be found in the SI. All images show maximum intensity projections of z-stacks. Scale bars: $10 \mu \mathrm{m}$.

3.4 N-YFP does not co-localize with microtubules. Vimentin and actin represent two of the three key cytoskeleton components, namely intermediated filaments (vimentin), the actin network and microtubules. To test for spatial correlation between the latter and $\mathrm{N}$ YFP we have performed immunofluorescence experiments, staining N-YFP transfected cells for tubulin (Figure 3). Both microtubule staining and N-YFP consistently showed tubular/fibrillar structures, which nonetheless showed only minor, if any, co-localization 
(Figure 3, cell \#1 and \#2). This held true even in the perinuclear region, where the microtubule organizing center (MTOC) and N-YFP formed pronounced, but independent cluster (Figure 3, cell \#2).

3.5 N-YFP puncta heavily associated with P-bodies. All hantaviruses are highly dependent on the cellular transcription machinery. An essential process called cap snatching involves cellular P-bodies (PB), which provide mRNA caps that serve as primers for the replication of the viral genome by the hantavirus RNA dependent RNA polymerase (RdRp) [6,8]. N proteins and RdRp have been proposed to be involved in that process. Here we sought to test whether N-YFP, in absence of other viral components, translocates to PBs, or if this process requires vRNAs, RdRp or viral glycoproteins. N-YFP transfected cells were stained for PBs using antibodies against the PB-resident protein Dcpa1. A noticeable correlation was found between PBs and larger N-YFP aggregates, but even more pronounced, with smaller N-YFP puncta (Figure 3B and SI Figure S3). PBs seemed to align with fibrillar N-YFP structures, whereas punctate structures showed high degrees of colocalization.

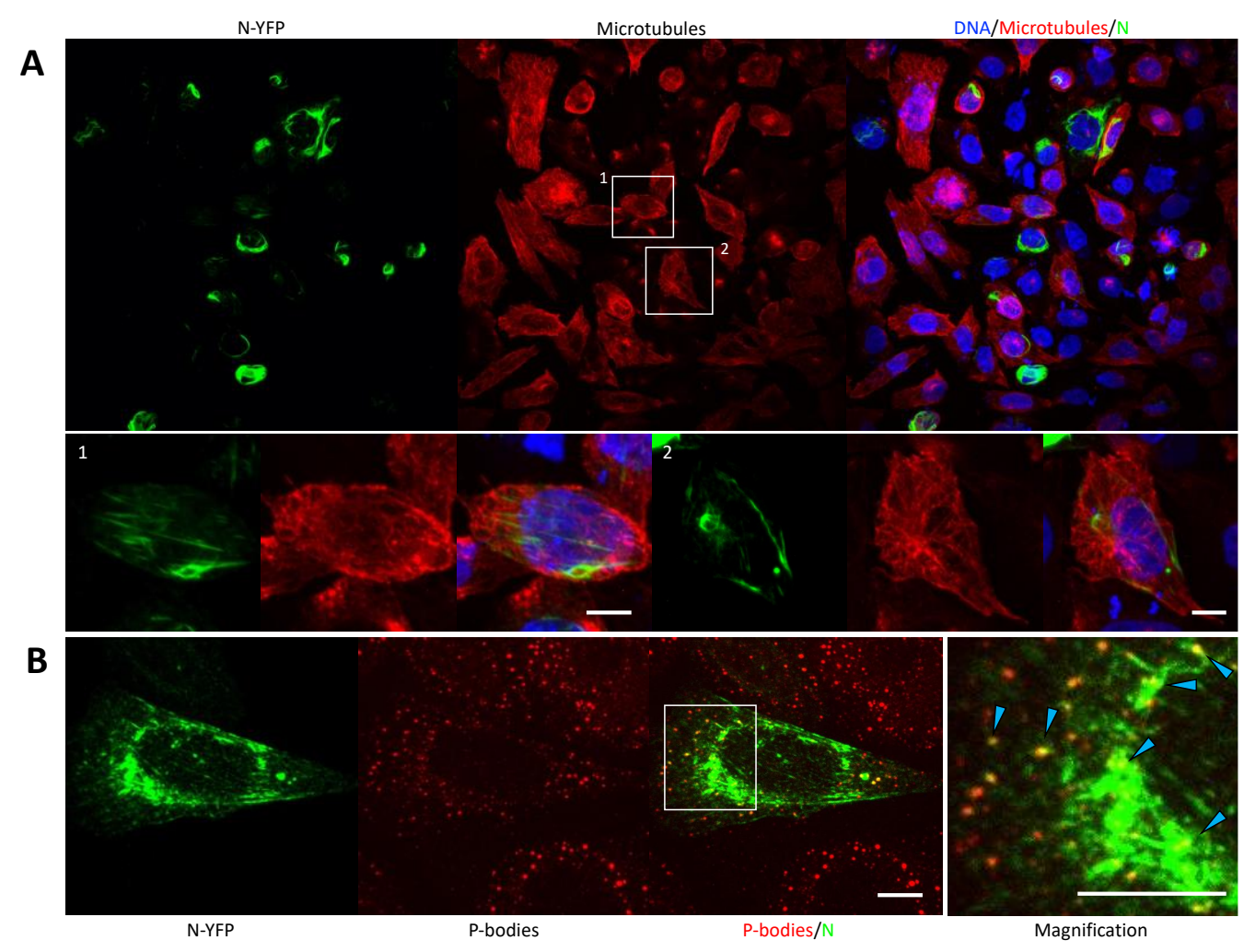

Figure 3: N-YFP co-localizes with P-bodies. (A) CHO-K1 cells were transfected with N-YFP and stained for microtubules by immunofluorescence. The two boxed cells are shown magnified in the lower panel. (B) CHO-K1 cells were transfected with N-YFP and stained for P-bodies by immunofluorescence. A magnification of the boxed area is shown on the right. Punctate patterns with clear co-localization of P-bodies and N-YFP are highlighted with arrows. All images show maximum intensity projections of z-stacks. Scale bars: $10 \mu \mathrm{m}$. Additional examples can be found in the SI.

3.6 Unsupervised, quantitative image analysis corroborates N-YFP co-localization with PBs, actin and vimentin. Our fluorescence microscopy images indicated a marked association of N-YFP with actin, vimentin and PBs, but not with microtubules. Initially, we have performed some image quantification of actin-stained cells by line-plot analyses. Now, in order to avoid any observer bias in our quantitative analysis we next performed automated image segmentation and quantification (SI Figure 4) using the cell profiler software package [17]. Pearson correlations were obtained for all above shown microscopy experiments, as well as an additional positive control staining of N-YFP transfected cells with a commercial N-protein antibody. This quantitative data analysis strongly supports 
our above-described observations, demonstrating that N-YFP significantly co-localizes with vimentin, PBs, and actin, but not with tubulin (Figure 4).

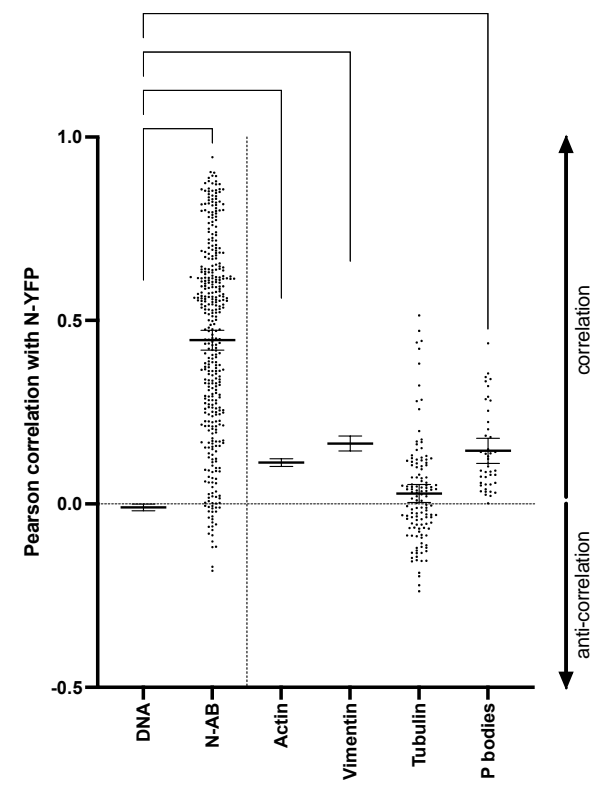

Figure 4: Quantitative automated image analysis reveals association of N-YFP with actin filaments, vimentin and P-bodies, but not Tubulin. Micrographs as shown in figures 1-3 were subjected to automated image analysis using cell profiler. Image segmentation examples are shown in the SI (Figure S4) and described in detail in the material and methods section. Bars show Pearson correlation of N-YFP with the markers as stated. Dots show individual cells $(n>20)$. DNA staining is utilized as a negative control staining assuming negligible N-YFP expression in the nucleus. N protein antibody staining $(\mathrm{N}-\mathrm{AB})$ was employed as a positive control. All bars show mean with SEM. Significance was tested using a one-way Analysis of variance (ANOVA) ${ }^{* * *} \mathrm{p} \leq 0.0001$.

3.7 N-YFP co-localizes with Gc and Gn. We have previously generated fluorescently tagged versions of both hantavirus glycoproteins, Gc and Gn [20]. Our recent work unequivocally demonstrated that upon co-expression these two proteins are highly enriched in the perinuclear region, whereas separately expressed, Gc and Gn show different localization patterns. We thus surmised that Gc and Gn are interacting in the endoplasmic reticulum, thus mutually promoting trafficking to the Golgi/ER-Golgi intermediate compartment (ERGIC) [20]. Now, we were interested to assess whether N-Turquoise, a cyanfluorescent version of N-YFP would equally co-localize with the Gc-mCherry and GnYFP. In fact, we observed strong spatial correlation in fluorescence microscopy images and found significant co-localization between all three proteins by automated image analysis. Noteworthily though, co-localization was significantly more pronounced between Gc and Gn than between N-Turquoise and either glycoprotein (Figure 5). 
A

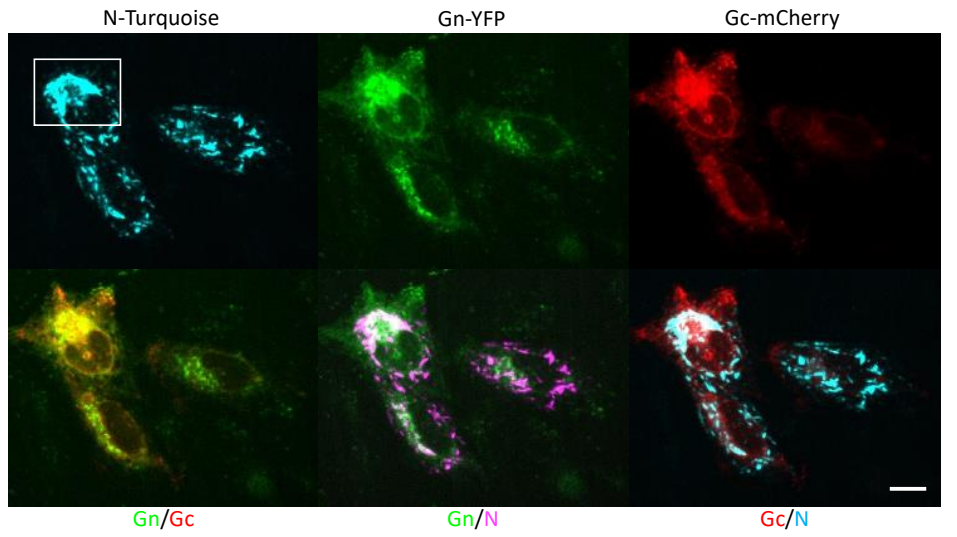

B
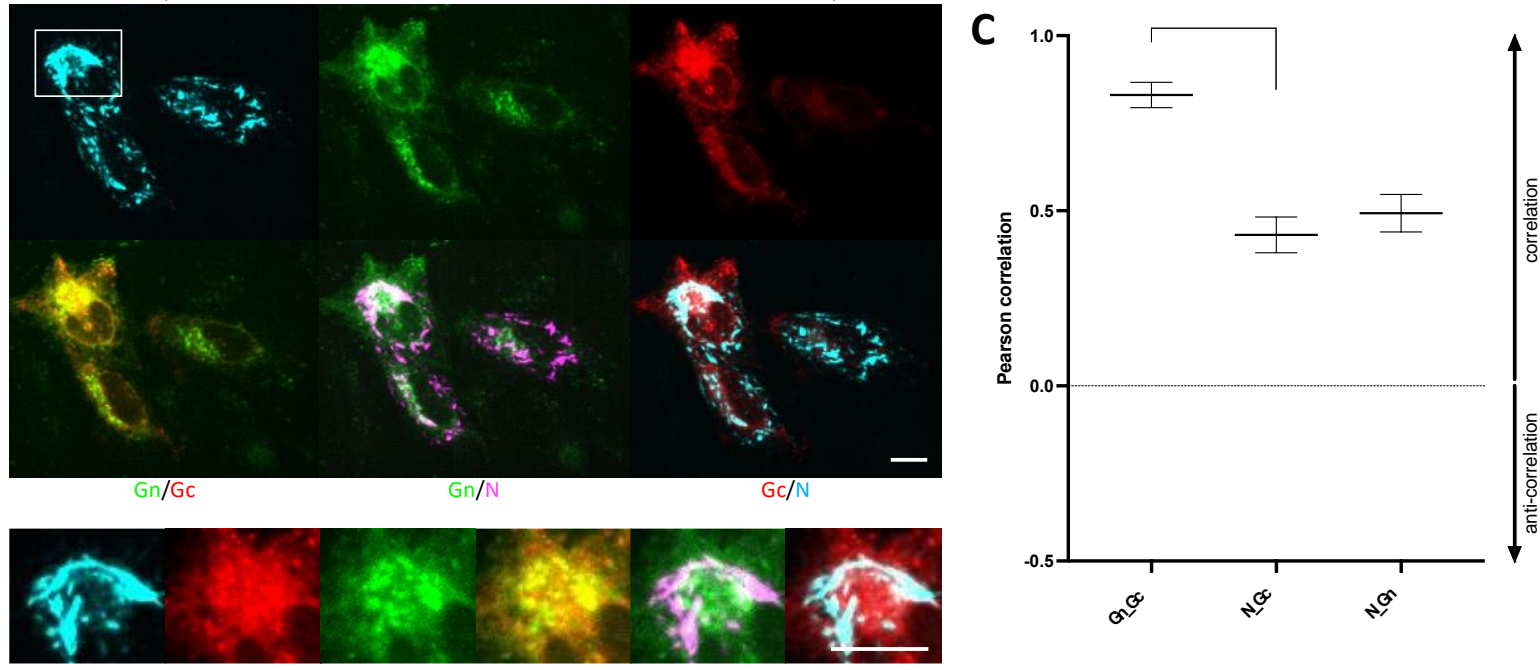

Figure 5: N-Turquoise co-localizes with fluorescently tagged Gc and Gn. (A) CHO-K1 cells were transfected with N-Turquoise (shown in cyan), Gn-YFP (green) and Gc-mCherry (red). (B) Magnification of the boxed region shown in A. Co-localization in overlay images appears orange (green and red), white (green and magenta, or red and cyan). Scale bars: $10 \mu \mathrm{m}$. Additional examples can be found in the SI. (C) Pearson correlation between proteins as stated in the bar chart were assessed by automated image analysis as described in Figure 4 . All bars show mean with SEM $(n=38)$. Significance was tested using a one-way Analysis of variance (ANOVA) ${ }^{* * *} \mathrm{p} \leq 0.0001$.

3.8 $\mathrm{N}$ protein multimerization is dose-dependent and independent of hantavirus glycoproteins. Hantavirus $\mathrm{N}$ protein has previously been reported to form dimers, trimers and high order multimers [11,24-26]. We employed Number and Brightness analysis (N\&B) to study N-YFP self-assembly in CHO-K1 cells. This fluorescence fluctuation spectroscopy technique determines the fluorescence intensity (molecular brightness) of individual protein complexes (molecular brightness), thus effectively assessing the oligomerization state of the protein under investigation in a live, single-cell context. We have previously utilized this method to assess oligomerization of both Gc and Gn $[18,20]$ and used it here to further characterize N-YFP. We also sought to test whether interactions between the hantavirus structural proteins affect oligomer assembly, thus monitoring N-YFP oligomerization in presence and absence of Gc and Gn (both fluorescently tagged). We focused on cells with relatively low expression levels of N-YFP in order to capture early oligomerization events and therefore the dynamics of the self-assembly process. Expectedly, when N-YFP was transfected alone, we found a marked correlation between overall N-YFP expression levels and N-YFP oligomerization, indicative of a high self-affinity of the viral protein (Figure 6A, B green dots and curves). N-YFP formed dimers, trimers and high order multimers, consisting of ca. ten individual proteins. Noteworthily, co-expression of either hantavirus glycoprotein did not significantly alter N-YFP self-assembly (Figure 6A, B red dots and curves), which showed a comparable dose-dependent self-association in absence and presence of either Gc or Gn. 

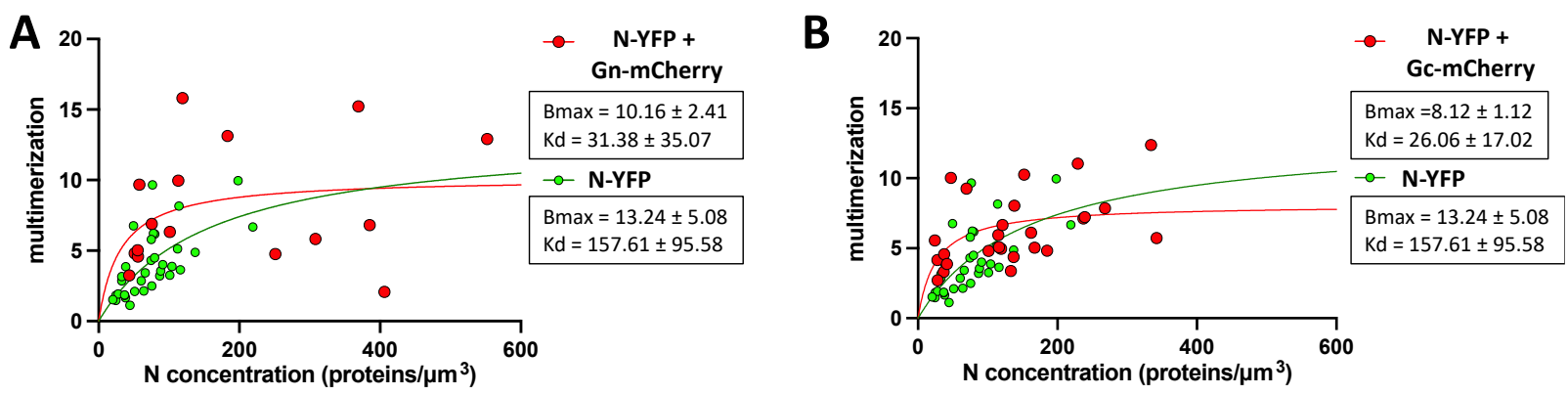

Figure 6: N-YFP forms high order multimers in presence and absence of Gc and Gn. N-YFP multimerization as a function of total protein concentration (in monomer units) in the absence and presence of (A) Gn-mCherry or (B) Gn-mCherry expressed in CHO-K1 cells. Each point in the graph represents the average NP multimerization and concentration within a region of interest in one $\mathrm{CHO}-\mathrm{K} 1$ cell. Solid lines show non-linear fits to a binding kinetic model $\left(\mathrm{Y}=\mathrm{Bmax}^{*} \mathrm{X} /(\mathrm{Kd}+\mathrm{X})\right)$ and dashed lines indicate $95 \%$ confidence intervals. Fit values with SEMs are shown in framed boxes.

\section{Discussion}

In this study we have investigated fluorescently labelled variants of the Puumalavirus nucleoprotein $(\mathrm{N})$. Two chimeric constructs, N-YFP and N-Turquoise were generated and analyzed in the rodent cell line $\mathrm{CHO}-\mathrm{K} 1$ to assess their intracellular time-resolved trafficking and localization, as well as co-localization with selected cellular proteins. In this context we specifically focused on the cytoskeleton proteins - tubulin (microtubular network), vimentin (intermediate filaments) and actin (actin network) - thus looking at all branches of this crucial morphological cellular structure. We have also assessed co-localization with P-bodies, which are critically involved in hantavirus replication cycles [8]. Finally, we performed co-transfection experiments with the viral glycoproteins Gc and Gn, both fluorescently labelled, observing significant co-localization of all three viral proteins, particularly in the perinuclear region. To be able to draw reliable objective conclusions from the microscopy study, we have conducted extensive automated image analyzes. We have focused on image segmentation using the cell profiler platform, developed by the Broad Institute since it enables rapid, unbiased cell identification and image quantification once a viable analysis pipeline was generated. We report pixel-by-pixel Pearson correlation, a measure of overall co-localization between two markers (fluorescent proteins and immunofluorescence staining), assessed on a single cell level, following automated image segmentation. Finally, we have conducted oligomerization studies, based on live, single-cell experiments using the Number and Brightness technique [27].

In our initial experiments we have observed that N-YFP, early after transfection, begins to form small, punctate clusters which rapidly coalesce first into pin-like structures of only a few micrometers length and ultimately into larger fibrillar aggregates that extend throughout large parts of the cytoplasm (Figure 1). Importantly, structures reminiscent of these N-YFP aggregates have been recently observed in persistent, long-term Tula virus [28] and Hantaan virus infections [15,16], but not during PUUV infections [16]. However, cluster formation is highly cell-type and context dependent [16] and it seems likely that oligomerization properties of $\mathrm{N}$ protein are shared across different Old world hantaviruses, in particular between phylogenetically closely related viruses such as Tula virus and PUUV [29]. N-YFP clustering typically starts and proceeds essentially in the perinuclear region and, importantly also in the cellular periphery. Perinuclear accumulation of viral proteins is considered a hallmark of hantavirus infections, reported for both New and Old world hantavirus species [30,31]. Peripheral localization and, in particular plasma membrane association, however, is rather controversial with only limited evidence for an involvement of the plasma membrane in hantavirus post-entry processes [32,33]. Our earlier studies indicated a significant, albeit low exposure of PUUV glycoproteins at the cell surface [20], suggesting that this process is not restricted to New world hantavirus as proposed previously $[33,34]$. 
General membrane association of $\mathrm{N}$ has been described early and is mediated through electrostatic interactions, likely by residues at the C-terminus of the protein [30]. We however surmise that the plasma membrane association we have observed in our livecell experiments is indicative of interactions with the cortical actin rather than direct interactions with the bilayer or surface exposed proteins. This hypothesis is supported by our immunofluorescence staining and image analysis results, showing marked co-localization between N-YFP and actin (Figure 2, Figure 4). Specifically filamentous N-YFP structures exhibit a remarkable similarity to and co-localization with actin filaments (Figure 2B). We also found significant co-localization of N-YFP with vimentin, but not with tubulin (Figure 2, Figure 3, Figure 4), indicative of specific interactions with either actin and vimentin directly or with other cellular proteins being associated with the respective cytoskeletal structures. Previous reports have highlighted the crucial function of the cytoskeleton during hantavirus infections $[5,9,15,35]$, however compelling evidence for direct interactions between individual hantavirus proteins and cytoskeleton proteins is scarce. Our data now strongly suggest that there could be a direct contact of $\mathrm{N}$ with actin and intermediate filaments, even in absence of any other viral components.

We furthermore observed significant co-localization between N-YFP and the P-body marker DCPa1 (Figure 3, Figure 4). P-bodies are believed to be responsible for storage and degradation of cellular RNA and have been implicated in hantavirus replication mechanisms by providing primers for viral mRNA synthesis in a process termed cap-snatching. Mir and colleagues have reported N P-body association for Sin Nombre virus, a New world hantavirus $[8,36]$, however to date, no Old world hantavirus has been investigated for such interactions. Of note, actin-interactions of $\mathrm{N}$ have been proposed to be involved in the trafficking of $\mathrm{N}$ to their P-body destination [15,35], underlining the key importance of actin for a successful hantavirus infection and replication.

We have also performed co-expression experiments with PUUV Gc and Gn, all fluorescently tagged. A strong co-localization was found between Gc or Gn with N, albeit less pronounced than co-localization between both glycoproteins. This is not surprising, given that Gc and Gn are membrane proteins that are derived from a common precursor whereas $\mathrm{N}$ is synthesized independently as a soluble, cytoplasmic protein. Nonetheless, co-localization between N-YFP and Gc/Gn could suggest a direct interaction between these viral proteins. However, our experimental approach cannot distinguish between physical interactions and co-enrichment in the same microenvironment (or cellular compartment).

Finally, we conducted N\&B experiments to study self-assembly of N-YFP clusters in presence and absence of Gc and Gn. We found a strong correlation between N-YFP oligomerization and overall expression levels, when N-YFP was transfected alone, suggesting a concentration-dependent cluster formation. We found monomers, dimer, trimers and larger multimers consisting of ca. 10 individual proteins. This is in agreement with previous publications, reporting evidence from mostly biochemical approaches, for a broad spectrum of $\mathrm{N}$ protein oligo- and multimers [11,24-26]. Importantly, the concentration dependency was not majorly altered in presence of either Gc or Gn, indicating that the viral glycoproteins do not interfere with N-YFP clustering. Future studies will have to investigate whether viral RNAs or other viral proteins contribute to N protein clustering, thus inducing the formation of even larger macromolecular complexes and ultimately complete viral particles.

Supplementary Materials: The following supporting information can be downloaded at: www.mdpi.com/xxx/s1, Figure S1: title; Table S1: title; Video S1: title.

Author Contributions: Conceptualization, R.S. and A.H.; methodology, R.W.W., H.S.S., A.K., C.S. S.C., R.S., A.H.; software, R.S., S.C.; validation, R.S. A.H., S.C., C.S.; formal analysis, R.W.W., H.S.S., A.K., R.S.; investigation, R.W.W., H.S.S., A.K.; resources, A.H., C.S., S.C., R.S.; data curation, R.S., A.H., C.S., S.C.; writing-original draft preparation, R.S.; writing-review and editing, R.W.W., H.S.S., A.K., C.S. S.C., R.S., A.H., D.H.K.; visualization, R.S.; supervision, D.H.K., R.S., A.H., C.S., S.C.; project administration, R.S., A.H., R.W.W.; funding acquisition, A.H., C.S., S.C., R.S. All authors have read and agreed to the published version of the manuscript. 
Funding: This work was supported by the German Research Foundation (DFG grant 407961559 to S.C.).

Institutional Review Board Statement: Not applicable.

Informed Consent Statement: Not applicable.

Data Availability Statement: Supplementary data are provided. Raw data are available upon request.

Acknowledgments: The bank vole (Myodes glareolus) lung cell line MGLU-R (CCLV-RIE 1304) was kindly provided by Prof. Rainer G. Ulrich (Friedrich-Loeffler-Institut, Greifswald - Insel Riems, Germany). We thank Dr. Thomas Korte and Ronny Bergmann for support.

Conflicts of Interest: The authors declare no conflict of interest.

\section{References}

1. Jonsson, C.B.; Figueiredo, L.T.M.; Vapalahti, O. A global perspective on hantavirus ecology, epidemiology, and disease. Clin. Microbiol. Rev. 2010, 23, 412-441, doi:10.1128/CMR.00062-09.

2. Kruger, D.H.; Figueiredo, L.T.M.; Song, J.-W.; Klempa, B. Hantaviruses-Globally emerging pathogens. J. Clin. Virol. 2015, 64, 128-136, doi:10.1016/j.jcv.2014.08.033.

3. Spiropoulou, C.F. Molecular Biology of Hantavirus Infection. In Bunyaviridae: Molecular and Cellular Biology.; Plyusnin, A; Elliott, R., Ed.; Caister Academic Press, 2011; pp. 41-60 ISBN 978-1-904455-90-5.

4. Mackow, E.R.; Gavrilovskaya, I.N.I. Hantavirus regulation of endothelial cell functions. Thromb. Haemost. 2009, 102, 103041, doi:10.1160/TH09-09-0640.

5. Bauherr, S.; Larsberg, F.; Petrich, A.; Sperber, H.S.; Klose-Grzelka, V.; Luckner, M.; Azab, W.; Schade, M.; Höfer, C.T.; Lehmann, M.J.; et al. Macropinocytosis and Clathrin-Dependent Endocytosis Play Pivotal Roles for the Infectious Entry of Puumala Virus. J. Virol. 2020, 94, 1-14, doi:10.1128/JVI.00184-20.

6. Vaheri, A.; Strandin, T.; Hepojoki, J.; Sironen, T.; Henttonen, H.; Mäkelä, S.; Mustonen, J. Uncovering the mysteries of hantavirus infections. Nat. Rev. Microbiol. 2013, 11, 539-50, doi:10.1038/nrmicro3066.

7. Torriani, G.; Mayor, J.; Zimmer, G.; Kunz, S.; Rothenberger, S.; Engler, O. Macropinocytosis contributes to hantavirus entry into human airway epithelial cells. Virology 2019, 531, 57-68.

8. Mir, M. a; Duran, W. a; Hjelle, B.L.; Ye, C.; Panganiban, a T. Storage of cellular 5' mRNA caps in P bodies for viral capsnatching. Proc. Natl. Acad. Sci. U. S. A. 2008, 105, 19294-9, doi:10.1073/pnas.0807211105.

9. Ravkov, E. V.; Nichol, S.T.; Peters, C.J.; Compans, R.W. Role of Actin Microfilaments in Black Creek Canal Virus Morphogenesis. J. Virol. 1998, 72, 2865-2870, doi:10.1128/jvi.72.4.2865-2870.1998.

10. Plyusnin, A.; Elliott, R. Bunyaviridae: Molecular and Cellular Biology; Caister Academic Press, 2011; ISBN 978-1-912530-52-6.

11. Reuter, M.; Krüger, D.H. The nucleocapsid protein of hantaviruses: much more than a genome-wrapping protein. Virus Genes 2018, 54, 5-16, doi:10.1007/s11262-017-1522-3.

12. Hepojoki, J.; Strandin, T.; Wang, H.; Vapalahti, O.; Vaheri, a; Lankinen, H. Cytoplasmic tails of hantavirus glycoproteins interact with the nucleocapsid protein. J. Gen. Virol. 2010, 91, 2341-50, doi:10.1099/vir.0.021006-0.

13. Strandin, T.; Hepojoki, J.; Wang, H.; Vaheri, A.; Lankinen, H. The cytoplasmic tail of hantavirus Gn glycoprotein interacts with RNA. Virology 2011, 418, 12-20, doi:10.1016/j.virol.2011.06.030.

14. Rowe, R.K.; Suszko, J.W.; Pekosz, A. Roles for the Recycling Endosome, Rab8, and Rab11 in hantavirus release from epithelial cells. Immunology 2009, 382, 239-249, doi:10.1016/j.virol.2008.09.021.Roles.

15. Ramanathan, H.N.; Jonsson, C.B. New and Old World hantaviruses differentially utilize host cytoskeletal components during their life cycles. Virology 2008, 374, 138-150, doi:10.1016/j.virol.2007.12.030.

16. Hägele, S.; Müller, A.; Nusshag, C.; Reiser, J.; Zeier, M.; Krautkrämer, E. Virus- and cell type-specific effects in orthohantavirus infection. Virus Res. 2019, 260, 102-113, doi:10.1016/j.virusres.2018.11.015.

17. Carpenter, A.E.; Jones, T.R.; Lamprecht, M.R.; Clarke, C.; Kang, I.H.; Friman, O.; Guertin, D. a; Chang, J.H.; Lindquist, R. a; 
Moffat, J.; et al. CellProfiler: image analysis software for identifying and quantifying cell phenotypes. Genome Biol. 2006, 7, R100, doi:10.1186/gb-2006-7-10-r100.

18. Petazzi, R.A.; Koikkarah, A.A.; Tischler, N.D.; Chiantia, S. Detection of Envelope Glycoprotein Assembly from Old World Hantaviruses in the Golgi Apparatus of Living Cells. J. Virol. 2021, 95, doi:10.1128/jvi.01238-20.

19. Digman, M.A.; Dalal, R.; Horwitz, A.F.; Gratton, E. Mapping the Number of Molecules and Brightness in the Laser Scanning Microscope. Biophys. J. 2008, 94, 2320-2332, doi:10.1529/biophysj.107.114645.

20. Sperber, H.S.H.S.; Welke, R.-W.R.-W.; Petazzi, R.A.R.A.; Bergmann, R.; Schade, M.; Shai, Y.; Chiantia, S.; Herrmann, A.; Schwarzer, R. Self-association and subcellular localization of Puumala hantavirus envelope proteins. Sci. Rep. 2019, 9, 707, doi:10.1038/s41598-018-36879-y.

21. Mayer, M.C.; Schauenburg, L.; Thompson-Steckel, G.; Dunsing, V.; Kaden, D.; Voigt, P.; Schaefer, M.; Chiantia, S.; Kennedy, T.E.; Multhaup, G. Amyloid precursor-like protein 1 (APLP1) exhibits stronger zinc-dependent neuronal adhesion than amyloid precursor protein and APLP2. J. Neurochem. 2016, 137, 266-276, doi:10.1111/jnc.13540.

22. Dunsing, V.; Chiantia, S. A Fluorescence Fluctuation Spectroscopy Assay of Protein-Protein Interactions at Cell-Cell Contacts. J. Vis. Exp. 2018, doi:10.3791/58582.

23. Binder, F.; Lenk, M.; Weber, S.; Stoek, F.; Dill, V.; Reiche, S.; Riebe, R.; Wernike, K.; Hoffmann, D.; Ziegler, U.; et al. Common vole (Microtus arvalis) and bank vole (Myodes glareolus) derived permanent cell lines differ in their susceptibility and replication kinetics of animal and zoonotic viruses. J. Virol. Methods 2019, 274, 113729, doi:10.1016/j.jviromet.2019.113729.

24. Alfadhli, A.; Love, Z.; Arvidson, B.; Seeds, J.; Willey, J.; Barklis, E. Hantavirus Nucleocapsid Protein Oligomerization. J. Virol. 2001, 75, 2019-2023, doi:10.1128/jvi.75.4.2019-2023.2001.

25. Alfadhli, A.; Steel, E.; Finlay, L.; Bächinger, H.P.; Barklis, E. Hantavirus nucleocapsid protein coiled-coil domains. J. Biol. Chem. 2002, 277, 27103-27108, doi:10.1074/jbc.M203395200.

26. Kaukinen, P.; Koistinen, V.; Vapalahti, O.; Vaheri, A.; Plyusnin, A. Interaction between molecules of hantavirus nucleocapsid protein. J. Gen. Virol. 2001, 82, 1845-1853, doi:10.1099/0022-1317-82-8-1845.

27. Chen, Y.; Johnson, J.; Macdonald, P.; Wu, B.; Mueller, J.D. Observing Protein Interactions and Their Stoichiometry in Living Cells by Brightness Analysis of Fluorescence Fluctuation Experiments. In Methods in enzymology; 2010; Vol. 472, pp. 345-363 ISBN 9780123749543.

28. Davies, K.A.; Chadwick, B.; Hewson, R.; Fontana, J.; Mankouri, J.; Barr, J.N. The RNA Replication Site of Tula Orthohantavirus Resides within a Remodelled Golgi Network. Cells 2020, 9, 1-18, doi:10.3390/cells9071569.

29. Hofmann, J.; Kramer, S.; Herrlinger, K.R.; Jeske, K.; Kuhns, M.; Weiss, S.; Ulrich, R.G.; Krüger, D.H. Tula Virus as Causative Agent of Hantavirus Disease in Immunocompetent Person, Germany. Emerg. Infect. Dis. 2021, 27, 1234-1237, doi:10.3201/eid2704.203996.

30. Ravkov, E. V; Compans, R.W. Hantavirus Nucleocapsid Protein Is Expressed as a Membrane- Associated Protein in the Perinuclear Region. J. Virol. 2001, 75, 1808-1815, doi:10.1128/JVI.75.4.1808.

31. Kallio-Kokko, H.; Leveelahti, R.; Brummer-Korvenkontio, M.; Lundkvist A; Vaheri, A.; Vapalahti, O. Human immune response to Puumala virus glycoproteins and nucleocapsid protein expressed in mammalian cells. J. Med. Virol. 2001, 65, 605-13, doi:10.1002/jmv.2079.

32. Ogino, M.; Yoshimatsu, K.; Ebihara, H.; Lee, B.; Okumura, M.; Arikawa, J.; Araki, K. Cell Fusion Activities of Hantaan Virus Envelope Glycoproteins Cell Fusion Activities of Hantaan Virus Envelope Glycoproteins. J. Virol. 2004, 78, 1077610782, doi:10.1128/JVI.78.19.10776.

33. Spiropoulou, C..; Goldsmith, C..; Shoemaker, T..; Peters, C..; Compans, R.. Sin nombre virus glycoprotein trafficking. Virology 2003, 308, 48-63, doi:10.1016/S0042-6822(02)00092-2.

34. Hepojoki, J.; Strandin, T.; Lankinen, H.; Vaheri, A. Hantavirus structure - molecular interactions behind the scene. J. Gen. Virol. 2012, 93, 1631-44, doi:10.1099/vir.0.042218-0. 
35. Ramanathan, H.N.; Chung, D.-H.; Plane, S.J.; Sztul, E.; Chu, Y.-K.; Guttieri, M.C.; McDowell, M.; Ali, G.; Jonsson, C.B. Dynein-dependent transport of the hantaan virus nucleocapsid protein to the endoplasmic reticulum-Golgi intermediate compartment. J. Virol. 2007, 81, 8634-47, doi:10.1128/JVI.00418-07.

36. Cheng, E.; Mir, M. a Signatures of host mRNA 5' terminus for efficient hantavirus cap snatching. J. Virol. 2012, 86, 10173-85, doi:10.1128/JVI.05560-11. 